\title{
Reproducible in-situ electrical biasing of resistive memory materials using piezo- controlled electrical contacts and chip based systems.
}

\author{
David Cooper and Matthew Bryan \\ Univ Grenoble Alpes, CEA, LETI, France
}

Piezo-based electrical biasing specimen holders, such as the Nanofactory and our recently acquired Hummingbird version use a movable contact that can be placed onto a desired region of interest with nm-scale resolution. Alternatively, chip based systems can be used where a device is electrically connected by using metal deposition in the focused ion beam tool such as the Protochips Aduro system. A working methodology has been developed for each type of specimen holder which will be discussed, as well as the advantages and disadvantages of using each type. These include methods to reduce leakage currents in the Ga implanted surface regions of the specimens as well as methods that can be used to provide ohmic contacts to reduce the effects of heating when applying voltages in-situ. To illustrate these developments a simple $\mathrm{p}-\mathrm{n}$ junction is studied where the electrical properties are well known.

We will present results from a $\mathrm{Pt} / \mathrm{SrTiO} 3 / \mathrm{Nb}: \mathrm{SrTiO} 3$ system is which is considered to be a model for the study of resistive memories [1]. This device can be switched electrically from a low resistive state (LRS) to a high resistive state (HRS). We will present aberration-corrected scanning (S)TEM and electron energy-loss spectroscopy (EELS) measurements of the composition and bonding that are obtained during cycling. In order to provide information about the different oxygen bonding states, Vertex Component Analysis (VCA) has been applied to the EELS data to provide abundance maps of the different components. It is thought that changes in the oxygen concentration around the electrodes are responsible for the switching and as such it is important to be able to visualize this movement.

Figure 1(a) shows a low magnification STEM image of an electrical probe being landed onto a device comprising (c) a Nb:doped SrTiO3 substrate (bottom electrode), a 18-nm-thick Sr-enriched SrTiO3 (Sr:STO) film and a 10$\mathrm{nm}$-thick Pt (top electrode). The device of nearly perfect crystalline structure has been electrically cycled multiple times (b) exhibiting so called eightwise or area switching. EELS spectra have been acquired of the region of interest in both high resistive (HRS) and low resistive (LRS) states. The Ti profiles shown in (d) that have been acquired from bottom to top across the insulateing STO region show that at the top of this region there is a change in the $\mathrm{L}_{3}$ edges which are linked to oxygen bonding. This can be seen from the reduction of the $\mathrm{t} 2 \mathrm{~g}$ edge and the shift of the Eg edge [3]. Figures 1(e) and (f) show maps of Ti-O bonds derived from the fine structure showing that for the LRS there is a high concentration of oxygen vacancies under the Pt electrode.

Figures 2(a) and (b) shows a similar specimen being attached to a chip type device and (c) and (d) show STEM images revealing many phase boundaries. This device should preferentially exhibit filamentary or counter eightwise switching due to the weak points in the structure. This is observed in Figure 2(e) as the device is cycled in-situ in where three decades of resistance switching are observed. The bright field STEM image in (f) shows that after switching some contrast is observed at the interface between the substrate and the active region. Figure 3(a) shows a BF STEM image of the region of interest. Averaged EELS spectra can be acquired for the (b) Ti and (c) O edges for the different electrical states. However, the complex structure of the devices means that it is more difficult to understand how they work. VCA has been applied to the data to provide maps of the different components of the spectra. (d) and (e) show maps of the different Ti states for the LRS and (f) and (g) for the HRS. The maps in (d) and (f) show the abundance of the spectra shown in (h) and the maps in (e) and (g) show the abundance of the spectra shown in (i) which corresponds to standard STO spectra with 4 sharp edges. 

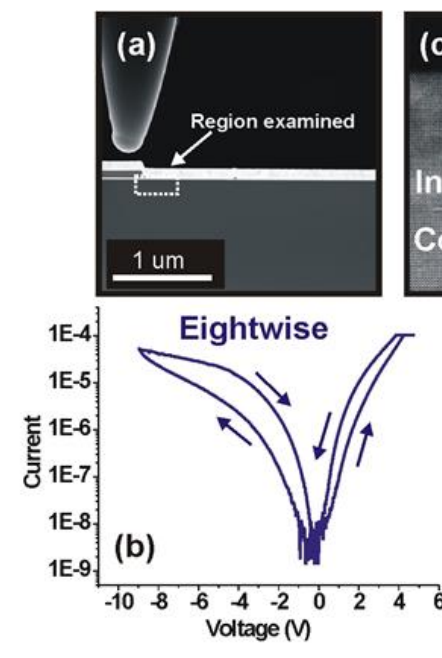

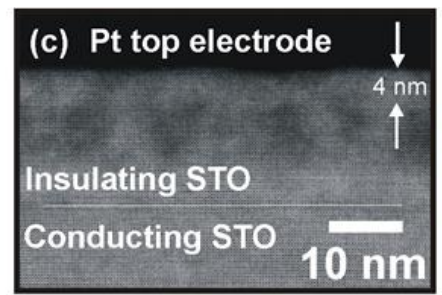

(d)

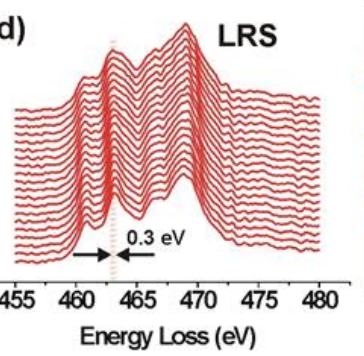

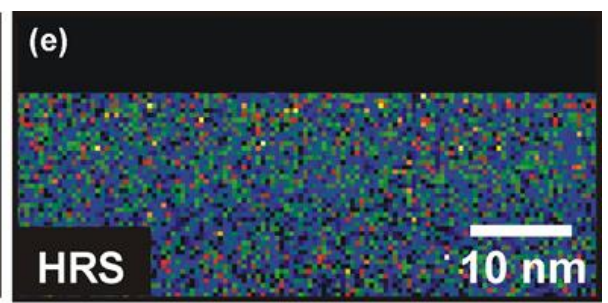

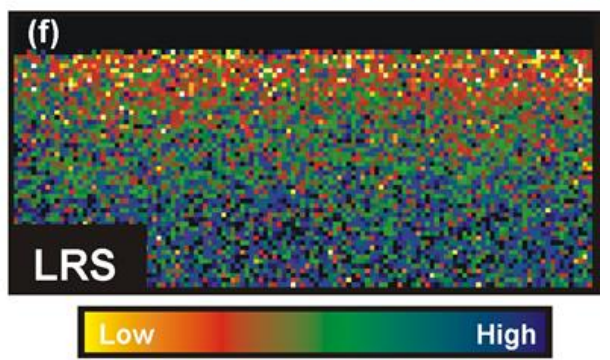

Figure 1. (a) A Pt/STO/Nb:STO stack is connected using a Nanofactory piezo holder (b) which is cycled in the TEM indicating area (eightwise) switching. (c) STEM images show that there is redistribution of material in the top $4 \mathrm{~nm}$ of the STO region. (d) Ti spectra show changes in the edges which can be linked to the Ti-O bonds or oxygen vacancies. (e) and (f) show Ti-O bonding maps for the HRS and LRS showing that there is a concentration of oxygen vacancies under the Pt top electrode for the LRS.
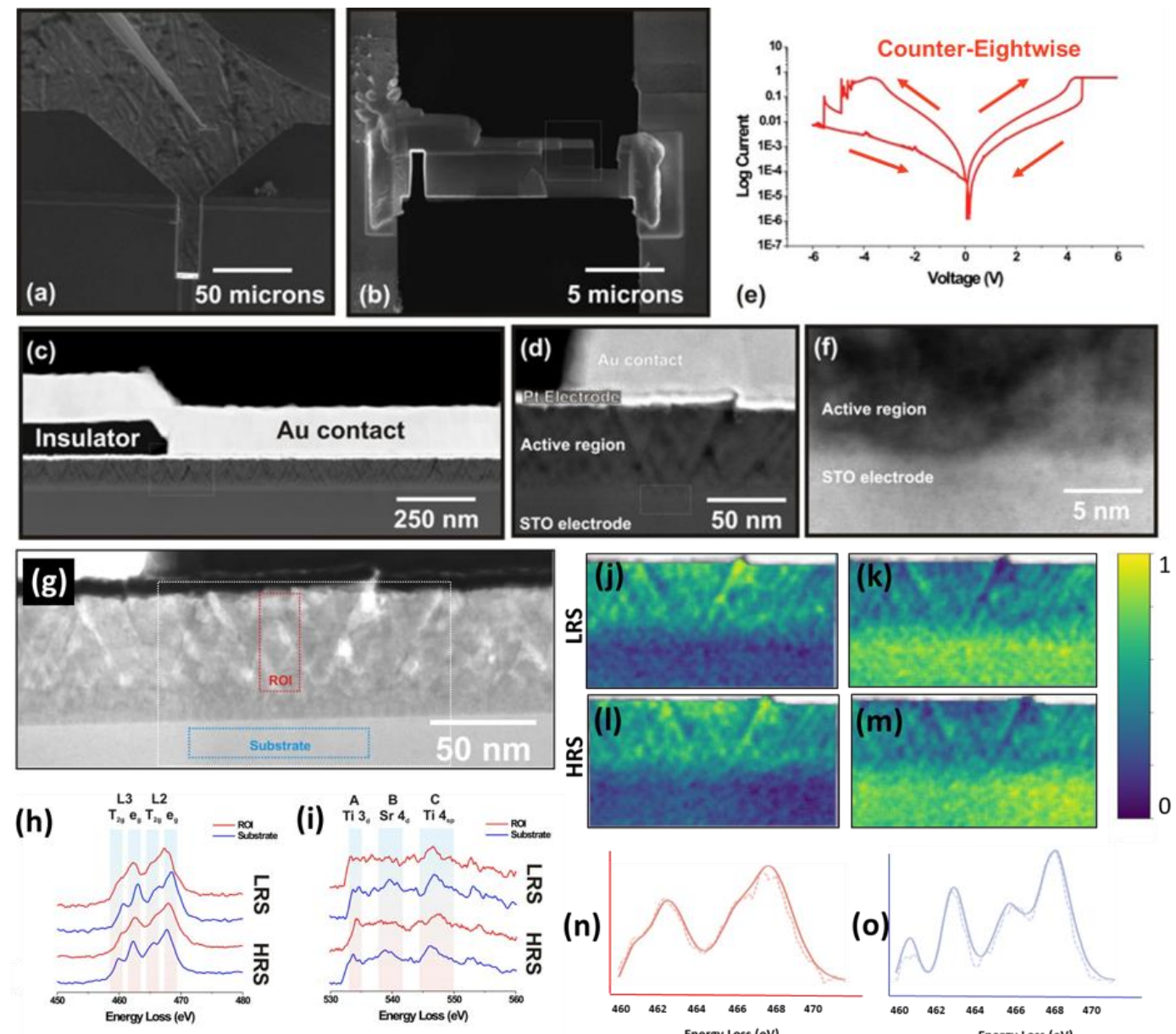

Figure 2. Figure 2: (a) The STO device is prepared by in-situ liftout in the FIB (b) and welded to a half biasing chip before (c) a thin region of the specimen is electrically connected. (d) Shows the local structure of region of the 
device showing phase boundries. (e) An I-V curve of the STO device showing counter-eightwise or filamentary switching. (f) Shows a high resolution bright field (BF) STEM image of the region indicated in (d). (g) a BF STEM image of the region of interest. Averaged EELS spectra for the (h) Ti and (i) O edges for the different electrical states. (j) and (k) show maps of the different Ti states for the LRS and (l) and (m) for the HRS obtained by VCA. The maps in (j) and (l) show the abundance of the TiO type spectra shown in (n) and the maps in (k) and (m) show the abundance of the spectra shown in (o) which corresponds to standard STO.

\section{References}

[1] R Waser and M. Aono, Nature Materials 6833 (2007)

[2] D. Cooper et al, Advanced Materials 291700212 (2017)

[3] D. A. Muller et al, Nature 430657 (2004)

[4] Acknowledgments: David Cooper thanks the European Research Council for funding the Starting Grant Holoview (Stg:506535). These experiments were performed on the platform Nanocharacterisation at Minatec (PFNC). 